\title{
Pectoralis muscle metastases from breast cancer in a young patient detected by automated breast ultrasound
}

\author{
Anca Ileana Ciurea ${ }^{1}$, Ioana Boca ${ }^{2}$, Liliana Rogojan ${ }^{2}$, Larisa Dorina Ciule², \\ Cristiana Augusta Ciortea ${ }^{2}$
}

${ }^{1}$ Department of Radiology, "Iuliu Hatieganu" University of Medicine and Pharmacy, ${ }^{2}$ Department of Radiology, Emergency County Hospital, Cluj-Napoca, Romania

\begin{abstract}
Metastases to the skeletal muscle from breast cancer represent an unusual and rare condition. We present the case of a 27-year-old female with left breast cancer (IDC NST G3) who underwent neoadjuvant chemotherapy followed by conservative surgery (sectorectomy and lymphadenectomy) and radiation therapy. Two months after the end of radiotherapy she presented with a $2 \mathrm{~mm}$ skin lesion and she was referred for a screening ultrasound. The screening automated breast ultrasound (ABUS) revealed local recurrence and pectoralis metastases, lesions evaluated also by magnetic resonance imaging. The diagnosis was confirmed by the ultrasound-guided biopsy.
\end{abstract}

Keywords: automated breast ultrasound, breast cancer, pectoralis metastases

\section{Introduction}

Early stage breast cancer metastasizes to regional lymph nodes. The most frequent sites of spreading in advanced stages are bones $(70 \%)$, lungs $(66 \%)$ and liver $(61 \%)[1]$.

Even though skeletal muscles are well vascularized, metastases from breast cancer represent an extremely rare condition. There are a few conditions that may affect the establishment of the tumor emboli in the muscles such as a variable blood flow due to contraction and relaxation of the striated fibers, mechanical destruction, the ability of skeletal muscle to remove lactic acid (which is involved in neovascularization) [2]. We present the case of a young patient with breast cancer and metastasis in the pectoralis muscle.

Received 23.10.2018 Accepted 20.12.2018

Med Ultrason

2019, Vol. 21, No 2, 200-203

Corresponding author: Ioana Boca, MD

Buna Ziua Street, no 39, bl E7D, sc 2, ap 84 Cluj-Napoca, Romania

E-mail: ioanaboca90@yahoo.com

Phone: +40756779095

\section{Case report}

A 27-year-old female presented with a palpable lump at 2.30 o'clock in the left breast. Past medical and family history was irrelevant. Ultrasound revealed a suspect hypoechoic, spiculated mass in the upper outer quadrant, so a $3 \mathrm{D}$ mammography was performed (fig $1 \mathrm{a}, \mathrm{b}$ ). The lesion was highly suspicious for malignancy on both examinations, therefore it was classified according to the Breast Imaging-Reporting and Data System (BI-RADS) as BI-RADS 5 and a percutaneous biopsy was taken. The pathological diagnosis was triple negative invasive ductal carcinoma (IDC NST Gr 3, ER 0\%, PR 0\%, HER2 -, Ki67 90\%).

Subsequently, the patient underwent neoadjuvant chemotherapy (4x Farmorubicin + Cyclophosphamide and 5x Docetaxel), conservative surgery (sectorectomy and lymphadenectomy) and radiation therapy.

Two months after the end of radiation she presented with a $2 \mathrm{~mm}$ skin lesion and she was referred for a screening automated breast ultrasound (ABUS). Two hypoechoic masses were detected: one at 2 o'clock, 53.1 $\mathrm{mm}$ from the nipple, cranial to the postsurgical scar (partially circumscribed) and the second in the intercos- 

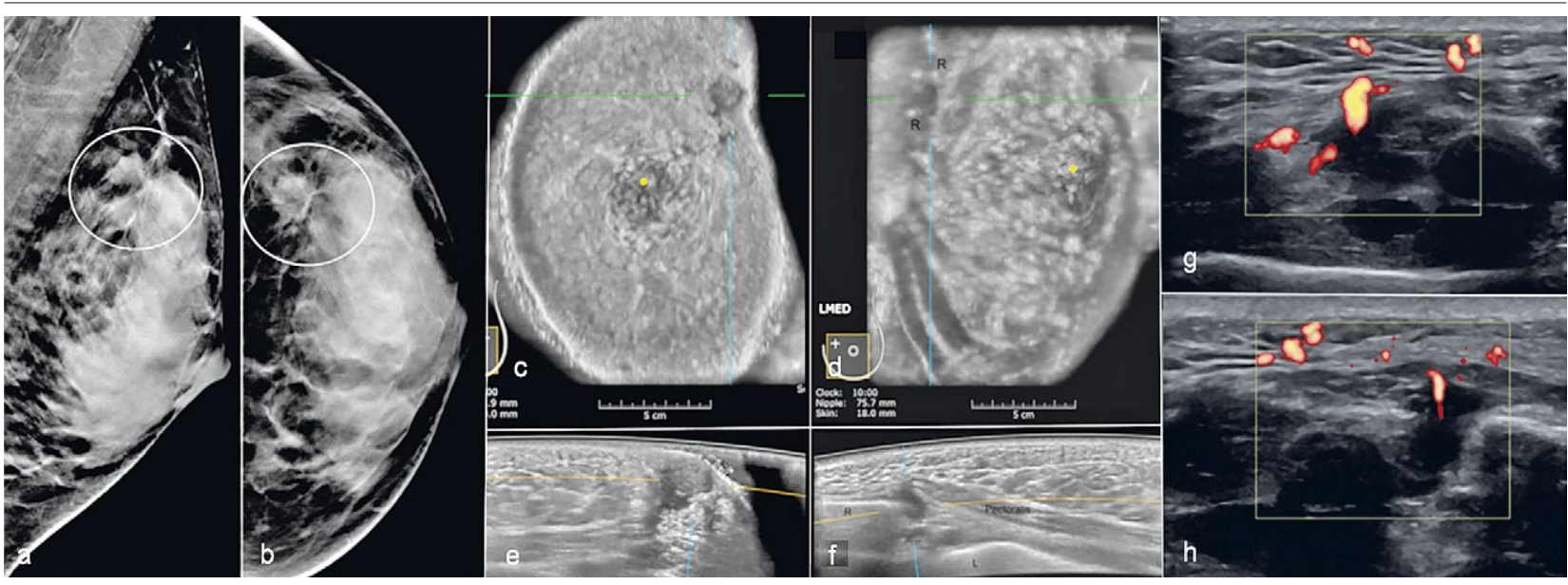

Fig 1. 3D left mammography: a) mediolateral oblique view; b) cranio-caudal view of an extremely dense breast reveals in the upper outer quadrant an irregular, spiculated mass with architectural distortion. Automated breast ultrasound acquisitions reveal a hypoechoic, partially circumscribed mass located at 2 o'clock, $53.1 \mathrm{~mm}$ from the nipple (yellow dot), cranial to the postsurgical scar (c) and d) and a hypoechoic, ill-defined mass located between two ribs (R), in a left intercostal space (e and f). Handheld ultrasound shows two hypoechoic, ill-defined and well-vascularized lesions ( $\mathrm{g}$ and $\mathrm{h}$ ).

tal space, parasternal, at 10 o'clock, $75.5 \mathrm{~mm}$ from the nipple (ill-defined) (Fig $1 \mathrm{c}-\mathrm{f})$. The examinations were completed with a handheld ultrasound (HHUS), which confirmed the presence of the lesions detected at ABUS and revealed a second lesion in the intercostal space (Fig $1 \mathrm{~g}, \mathrm{~h}$ ). The appearance of the lesions raised the suspicion of local recurrence and pectoralis metastases.

The contrast-enhanced breast magnetic resonance imaging (MRI) found that all lesions had a malignant morphology and behavior (Fig 2 a-d). The diagnosis was confirmed by the ultrasound-guided biopsy of the pectoralis lesions.

The patient started chemotherapy. After a week she underwent a contrast-enhanced computed tomography $(\mathrm{CT})$, which revealed mediastinal, supraclavicular and left axillary lymph node metastases, left pleural metastasis and left pleural effusion (Fig $2 \mathrm{e}-\mathrm{h}$ ).

Two months later the patient's general condition had worsened and she was hospitalized for acute respiratory failure with bilateral massive pleural effusions, pericardial effusion and multiple bones and subcutaneous metastases with unfavorable evolution to death.

\section{Discussions}

Breast cancer represents the most frequent type of cancer in women and the second cause of death due to cancer (after lung cancer), with a mortality rate of $2.6 \%$ [3]. Five molecular subtypes of breast cancer are recognized and triple negative subtype (ER- PR- HER2-) is one of them. It constitutes an aggressive form with a high

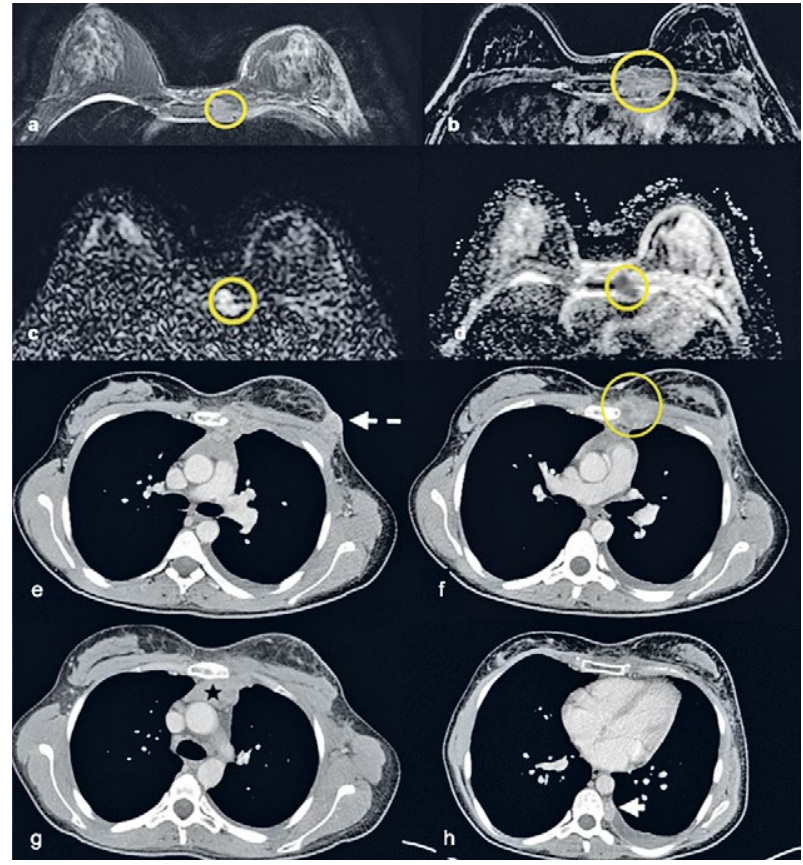

Fig 2. Magnetic resonance imaging found the suspect lesions located in the left pectoralis muscle, isointense on T2 (a), with pathological contrast enhancement (b), restriction on diffusionweighted imaging (c) and high apparent diffusion coefficient values (d). At contrast-enhanced CT, venous phase, axial sections homogeneous enhancement of a subcutaneous mass (e - white dotted arrow), inhomogeneous enhancement of the pectoralis muscle lesions and a small cutaneous lesion representing a permeation nodule (f - yellow circle), mediastinal lymph node metastases ( $g$ - black star), homogeneous enhancement of a left pleural lesion (white arrow) associated with left pleural effusion $(\mathrm{h})$ were described. 
risk of recurrence and an increased risk of death within 2 years of diagnosis [4].

$\mathrm{Ki}-67$ is a biomarker of tumor proliferation and a prognostic factor in primary breast cancer. Tashima et al established the value of $20 \%$ as being the optimal cutoff point for Ki-67 index in their study for neoadjuvant chemotherapy; the mean value in the triple negative tumor was $51.9 \%$ [5]. Unfortunately, our patient had a triple negative lesion, with a Ki-67 of $90 \%$, which lead to fulminant worsening of general condition and death.

In advanced disease stages, the risk of developing bone metastases is higher in cases with multiple lymph node metastases, HER2 positive or triple negative breast cancer subtypes [6]. On the other hand, skeletal muscle metastases (SMM) are uncommon. Most of the knowledge regarding this pathology has been achieved through multiple published case reports. In the vast majority of situations, SMM remain asymptomatic (as in our case) and only in a few cases may present as local swelling, pain or muscle impairment [2]. The imaging findings may vary from round/oval lesion with homogeneous enhancement or abscess-like lesion to diffuse infiltrating aspect with inhomogeneous enhancement, multiple intramuscular calcifications or even intramuscular bleeding [7] and differential diagnosis is required $[8,9]$.

To the best of our knowledge, in the literature there are about 39 cases of SMM from breast cancer, but none of them in the pectoralis muscle. Some of these cases have been reviewed from the literature or have come from retrospective studies [2,7,10-12] while ten of them have been published as individual case reports $[8,11$, 13-19].

The detection rate of ABUS is $97 \%$ in for lesions larger than $11 \mathrm{~mm}$. The diagnostic performance is similar to that of HHUS except for cases of a multifocal disease in which ABUS seemed to outperform HHUS [20,21]. In our patient, ABUS not only detected the local recurrence but also drew attention to the ill-defined, hypoechoic lesions located deep in the left intercostal space.

\section{Conclusions}

In conclusion: breast cancer in young patients is usually aggressive and can have a bad prognosis. ABUS represents a new screening technique that can be successfully used for detections and follow-up in young patients or patients with dense breast tissue.

\section{References}

1. Leong SP, Cady B, Jablons DM, et al. Clinical patterns of metastasis. Cancer Metastasis Rev 2006;25:221-232.
2. Khandelwal AR, Takalkar AM, Lilien DL, Ravi A. Skeletal Muscle Metastases on FDG PET/CT Imaging. Clin Nucl Med 2012;575-579.

3. American Cancer Society. Available at: https://www.cancer.org/about-us.html.

4. Lin NU, Vanderplas A, Hughes ME, et al. Clinicopathologic features, patterns of recurrence, and survival among women with triple-negative breast cancer in the National Comprehensive Cancer Network. Cancer 2012;118:54635472.

5. Tashima R, Nishimura R, Osako T, et al. Evaluation of an Optimal Cut-Off Point for the Ki-67 Index as a Prognostic Factor in Primary Breast Cancer: A Retrospective Study. PLoS One 2015;10:e0119565.

6. Yamashiro H, Takada M, Nakatani E, et al. Prevalence and risk factors of bone metastasis and skeletal related events in patients with primary breast cancer in Japan. Int J Clin Oncol 2014;19:852-862.

7. Surov A, Hainz M, Holzhausen HJ, et al. Skeletal muscle metastases: primary tumours, prevalence, and radiological features. Eur Radiol 2010;20:649-658.

8. Cho KE, Son EJ, Kim JA, et al. Metastasis of Breast Carcinoma to Intercostal Muscle Detected by Breast MRI: A Case Report. J Korean Soc Radiol 2010;63:391-394.

9. Ciurea A, Dudea SM, Lebovici A, Fodor L, Crisan D. Diffuse angiomatosis of the breast-sonographic appearance. J Clin Ultrasound 2014;42:498-501.

10. Emmering J, Vogel WV, Stokkel MP. Intramuscular metastases on FDG PET-CT: a review of the literature. Nucle Med Commun 2012;33:117-120.

11. Ogiya A, Takahashi K, Sato M, et al. Metastatic breast carcinoma of the abdominal wall muscle: a case report. Breast Cancer 2015;22:206-209.

12. Wiggins RE Jr, Byrne SF. Metastatic tumor to the extraocular muscles: Report of 5 cases. J AAPOS 2012;16:489-491.

13. Kim YW, Seo KJ, Lee SL, et al. Skeletal muscle metastases from breast cancer: two case reports. J Breast Cancer 2013;16:117-121.

14. Bhattacharjee A, Rassell M, Hasan MN, Debnath BC. Abdominal wall metastasis of carcinoma breast: a case report. Bangladesh Med J 2015;44:172-174.

15. Kouvaris JR, Gkongkou PV, Papadimitriou CA, et al. Bilateral metastases to extraocular muscles from lobular breast carcinoma. Onkologie 2008;31:387-389.

16. Murthy R, Gupta A, Hegde S, Honavar SG. Bilateral multiple extraocular muscle metastasis from breast carcinoma. Indian J Ophthalmol 2011;59:381-382.

17. Salemis NS. Skeletal muscle metastasis from breast cancer: management and literature review. Breast Dis 2015;35:3740.

18. Gyorffy J, Philbrick SM, Bersabe AR, et al. A Unique Case of Muscle-Invasive Metastatic Breast Cancer Mimicking Myositis. Case Rep Oncol Med 2017;2017:2648296.

19. Khan NA, Morlese J, Khan A. Radiological foresight: a rare case of breast cancer metastases to the extraocular muscles. BMJ Case Rep 2015 Jul 15. doi:10.1136/bcr-2015211264. 
Med Ultrason 2019; 21(2): 200-203 203

20. Rella R, Belli P, Giuliani M, et al. Automated Breast Ultrasonography (ABUS) in the Screening and Diagnostic Setting: Indications and Practical Usage. Acad Radiol 2018;25:1457-1470.
21. Wang HY, Jiang YX, Zhu QL, et al. Differentiation of benign and malignant breast lesions: a comparison between automatically generated breast volume scans and handheld ultrasound examinations. Eur J Radiol 2012;81:3190-3200. 